\title{
Evaluation of Tri-axial accelerometery data of falls for elderly through smart phone
}

\author{
Golenur B. Huq, Jim Basilakis, Anthony Maeder \\ School of Computing, Engineering and Mathematics \\ Western Sydney University \\ Locked Bag 1797, Penrith 2751, New South Wales, Australia \\ g.huq@uws.edu.au; j.basilakis@uws.edu.au; a.maeder@uws.edu.au
}

\begin{abstract}
As the world population ages, falls among the elderly are becoming a significant burden on healthcare. Fall prevention programs provide solutions for alleviating this burden. Such programs can be supported through monitoring of the elderly with tri-axial accelerometer sensors and mobile technology in order to detect falls and ensure individuals receive rapid care. A six-month pilot program was undertaken that involved recording tri-axial accelerometer data from mobile phones designed to be worn and used by independent community-dwelling elderly individuals. Fall data gained through this pilot program has been analysed in order to determine the quality of data recorded and the feasibility of constructing a threshold based fall detection algorithm from this data. Issues are found with the sample rate and range of the recorded data. Despite this, fall detection of acceptable quality is found to be plausible through measurement of changes in posture.
\end{abstract}

Keywords: Elderly, smart phone, tri-axial accelerometer data.

\section{Introduction}

Falls are a major health problem globally, with an estimated 424,000 fatal falls occurring each year. This makes falls the second highest cause of accidental or unintentional death due to injury, after injuries caused by traffic accidents. However, not all falls are fatal, with the vast majority being non-fatal. 37.3 million non-fatal falls serious enough to require medical attention are estimated to occur every year (World Health Organization, 2012).

Death rates due to falls are highest among adults over the age of 60. Non-fatal falls impact the elderly significantly as well. For example, in the United States, $20-30 \%$ of older people who fall suffer moderate to severe injuries such as bruises, hip fractures, or head trauma. This risk level is likely due to physical, sensory and cognitive changes associated with aging, in combination with environments that are not adapted for an aging population. Falls among the elderly place a significant cost on the healthcare system as well, with each fall injury among the elderly estimated to cost on

Permission to make digital or hard copies of all or part of this work for personal or classroom use is granted without fee provided that copies are not made or distributed for profit or commercial a dvantage a nd that copies bear this notice and the full citation on the first p age. To copy otherwise, to republish, to post on servers or to redistribute to lists, requires prior specific permission and/or a fee.

ACE' 16 Canberra, ACT Australia

Copyright 2016 ACM 978-1-4503-4042-7/16/02 ...\$15.00

http://dx.doi.org/10.1145/2843043.2843385 average \$1049 in Australia (World Health Organization, 2012). The cost placed on health systems due to falls among the elderly will increase as the world's population ages, with 2 billion individuals expected to be above the age of 60 by the year 2050 (World Health Organization, 2013). Within the Australian Capital Territory (ACT), $10 \%$ of the overall population is comprised of elderly individuals over the age of 65. About one third of the elderly in the ACT sustain a fall every year. 3\% of these fallers require hospitalisation while $0.02 \%$ die. A recent study found that falls made up $31 \%$ of injury related hospital separations, two thirds of which are comprised of the elderly. (Guest, Halliday, Kee, O'Brien, \& O'Donoughue, 2010) This underlines the necessity of implementing effective methods of fall prevention.

In order to reduce the risk of falls amongst the elderly, implementing multidisciplinary fall intervention programs are essential. Such intervention programs should include components such as i) medical assessment, ii) home safety checks and advice, iii) monitoring of prescription medications, iv) environmental changes, v) tailored exercise and physical activity, vi) training in transfer skills and gait; vii) assessment of readiness to change behavior; and vii) referral of clients to health-care professionals (World Health Organization, 2007). Monitoring of the elderly using tri-axial accelerometer sensors and mobile technology can be used to support these fall intervention programs.

To investigate minimizing falls risk and the impact of falls, Anglicare (in ACT) in collaboration with the University of Western Sydney (UWS), and Mediinspect, a Czech Republic technology company, has undertaken a six-month pilot program involving taking recordings of tri-axial accelerometer readings from mobile phones designed to be worn and used by elderly persons who live independently in the community.

The pilot program was designed to investigate the properties of tri-accelerometer recordings whilst monitoring the elderly with a high risk of falls. This would allow assessment of the feasibility of detecting falls or changes in gait patterns in order to alert a call centre that further support is required for the individual. Participants could also use the technology to manually alert their carers of emergency fall situations. The technology was also capable of identifying its GPS location to assist those who may be at risk of wandering and disorientation, but this capability was not assessed during the trial.

This paper describes the technology used and the pilot trial methodology. This is followed by presentation of the initial findings, considering also some of the clinical 
implications. The data quality and feasibility of constructing a threshold-based falls detection algorithm is evaluated through analysis of the accelerometer data.

\section{Related work}

A number of studies have been conducted on monitoring falls using tri-axial accelerometer sensors, including those contained within mobile phones. Kangas et al determined that the optimal accelerometer position for fall detection is the waist (Kangas, Konttila, Winblad, \& Jämsä, 2007). This allows inbuilt phone accelerometers to be used for fall detection as long as phones are stored near the waist, such as on a pouch, or in a pocket.

Several studies examine simulated falls within lab environments (Dai, Bai, Yang, \& Shen, 2010), (Jia, 2009), (Boyle \& Karunanithi, 2008), (Bourke, O'Brien, \& Lyons, 2007), (Pérolle, 2006) (Zhao, Chen, Wang, \& Chen, 2012) (Kangas M. , Vikman, Wiklander, Lindgren, Nyberg, \& Jämsä, 2009) (Karantonis, Narayanan, Mathie, Lovell, \& Celler, 2006) (Nyan, Tay, Seah, \& Sitoh, 2006) (Dumitrache \& Pasca, 2013) (Jantaraprim, Phukpattaranont, Limsakul, \& Wongkittisuksa, 2010). Virtually all of these studies performed tests on young test subjects, despite Bourke et al (2007) postulating that fall signals from young test subjects in a simulated environment will be different to real world fall signals gained from the elderly. The algorithms developed by these studies are largely threshold-based, using acceleration sum vector, vertical velocity, or changes in posture angle as features. Kerdegari et al presented a fall detection algorithm based on machine learning, but did not achieve results of sufficient quality, only reaching an accuracy of $90.15 \%$ (Kerdegari, Samsudin, Ramli, \& Mokaram, 2012).

Bianchi et al and Sorvala et al have suggested improving fall detection through use of additional sensors, such as barometers to detect pressure altitude, and gyroscopes to more reliably detect changes in posture (Bianchi, Redmond, Narayanan, Cerutti, \& Lovell, 2010) (Sorvala, Alasaarela, Sorvoja, \& Myllyla, 2012).

A study has also been completed on real world falls involving older people over the age of 65 (Bagalà, et al., 2012). The study evaluated the performance of fall detection algorithms that applied to the database of 29 'real world' falls as a part of European project SensAction-AAL. Algorithms based on waist or trunk accelerometer measurements were investigated, and it was found that algorithms constructed based on data gained from simulated falls were not as effective when applied to detect real world falls. Kangas and et al also compared 5 'real world' falls in the elderly with experimental falls from middle-aged people. They found that the signals from real-life falls had similar features to intentional falls but some of the detected falls characteristics were not detectable in acceleration signals from real life falls (Kangas M. , Vikman, Nyberg, Korpelainen, Lindblom, \& Jämsä, 2012). Another study (Klenk, et al., 2011) found significant difference between simulated falls and real-world falls that involved falling backwards. Accelerometer signals obtained through waist mounted tri-axial accelerometers were compared in these studies. In general there is a lack of studies that involve assessment of real falls amongst the elderly using tri-axial accelerometer data. Despite how relatively common falls are among the elderly, recording real world fall data remains difficult and time consuming (Bagalà, et al., 2012).

\section{Technology}

\subsection{Smart phones}

The tri-axial accelerometer recordings in the pilot were collected using a smart phone (Samsung i555, see Figure 1), designed for the elderly, living independently in the community. Smart phones were equipped with the Android 2.2 Operating System which have tri-axial accelerometer sensor capabilities (Jia, 2009) to detect falls and changes in gait. The inbuilt tri-accelerometers were able to record acceleration data at $16.67 \mathrm{~Hz}(1000$ data points per minute) within the range of $\pm 2 \mathrm{~g}$. The smart phones were installed with the Mediiware InspectLife app to broadcast tri-axial accelerometer event data to the InspectLife Surveillance Web system and raw data to an associated Data Server.

The InspectLife application installed in the smart phones has the capability to send an 'Alert' to a call centre if a participant becomes unsteady or has sustained a fall, so support can be provided. Participants could also use the technology to manually send alerts by sending an SMS to their carers.

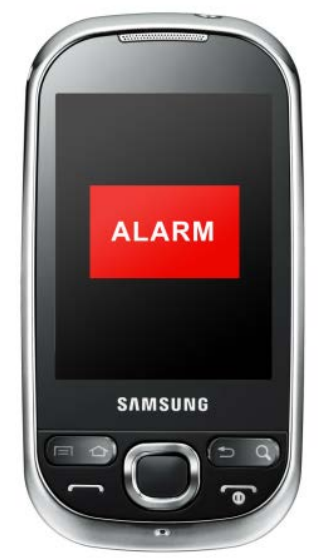

Figure 1: Mobile device (Samsung Smartphone)

\subsection{System description}

The overall system used for the pilot program includes two central repository systems:

a) Raw Movement Data System (RMDS): This system houses raw tri-axial accelerometer data from each smart phone. The size of the output data of the smart phone is approximately $4 \mathrm{MB} / \mathrm{h} /$ user when continuous raw movement data is measured, made up of 20-second cycles of raw data packets each of about $25 \mathrm{~KB}$ in size.

b) InspectLife Surveillance Web System (ISWS): This system includes a web browser and houses continuous surveillance data for $3 \mathrm{~GB}$ per month per 100 users. The output data of the smart phone for ISWS is $40 \mathrm{~KB} / \mathrm{h} / \mathrm{user}$ 


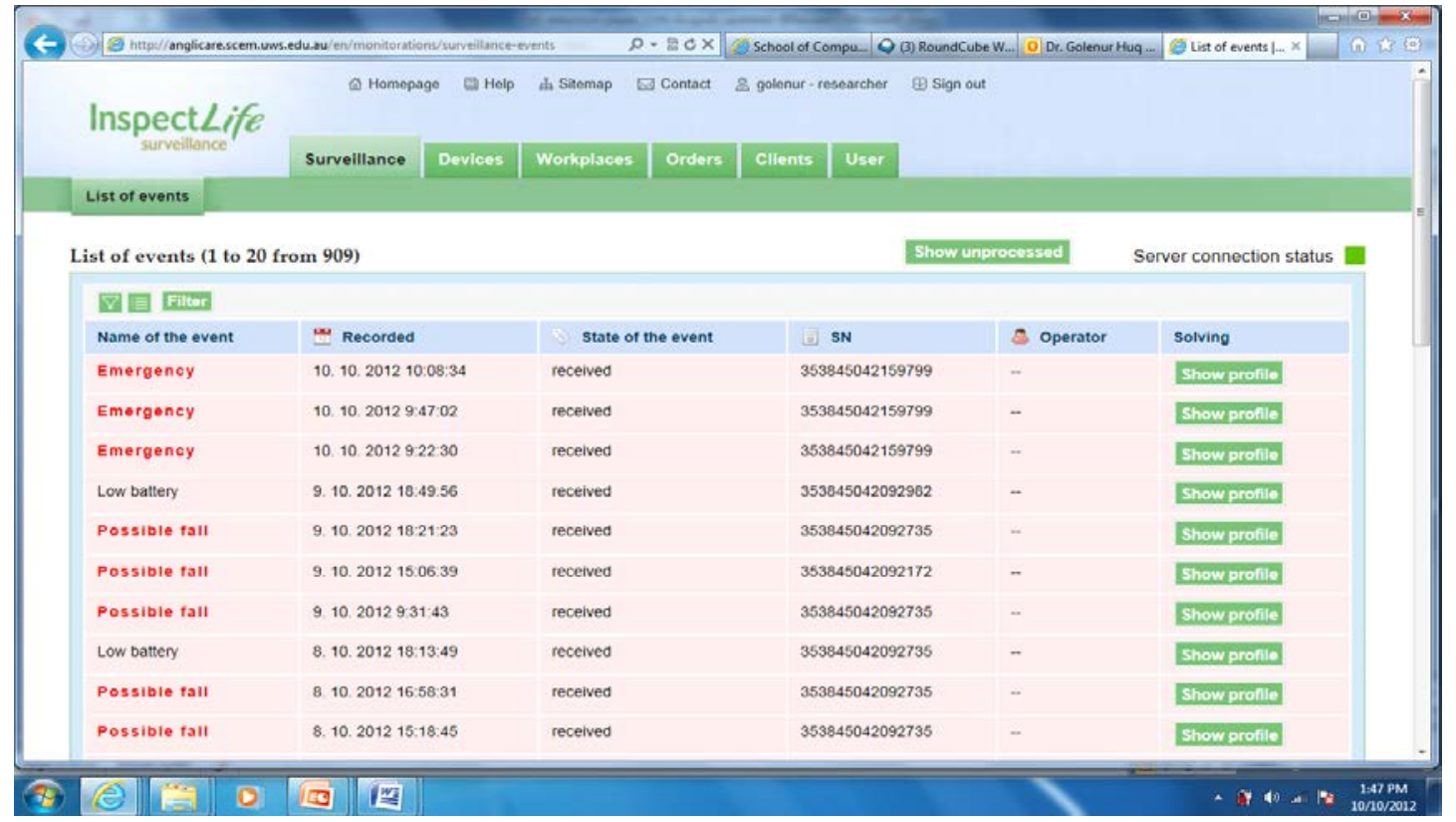

Figure 2: InspectLife Surveillance web system (ISWS)

and three types of data packets. They are i) 'Emergency' packets triggered by users/clients by pressing the "ALARM" (see Figure 1) from the smart phone; ii) 'ALIVE' packets are sent in every 10 minutes stored in RMDS; 'ALIVE' packets are those when device is active or in use, was set threshold to $10 \mathrm{~m} / \mathrm{s}^{2}$ used only for inactivity alarms; and move threshold was set to $15 \mathrm{~m} / \mathrm{s}^{2}$ for raw data recording initiation; and iii) 'SHAKE' packets that triggered by big movement which are captured in ISWS as 'Possible Fall'. This 'SHAKE' packets are enabled when the sum vector exceeds a set threshold that was set to $27 \mathrm{~m} / \mathrm{s}^{2}$ (Freescale Semiconductor, 2013).

\subsection{How the ISWS works}

If the smart phone detects a fall, a message is sent from the device that the ISWS captures as a 'Possible Fall'. A participant may also press an "ALARM" button on the smart phone manually if he/she has experienced a fall or some other crisis event. This triggers a message to the InspectLife web system, which displays an "Emergency" event as well as sends a text message to an operator, for immediate notification. This allows an operator to monitor patient falls and report to emergency services, family members or care-givers as necessary.

The InspectLife web system consists of a Surveillance page, Device page, Workplace page, Orders page, and a Client and User page (Figure 2). The Surveillance page contains a list of relevant events requiring notification such as a 'Possible Fall' as detected by the smart phone device, 'Emergency' events triggered by pressing the 'ALARM' button, as well as 'Low battery' and 'Lost connection' indicators. If an operator were notified about a possible fall, then he/she would contact the particular client to confirm that a fall has occurred and take action as necessary. Whether a fall has occurred or not is recorded at the scene by carers. Clinical notes about the patient relating to the event can be entered in the web sites that can be shared across a patient's care-givers as required. The ISWS also includes profile details of the participants, including their past clinical history and medications as well as other important information about registered carers and contact information. Device page also includes some device parameters for example, movement threshold, raw movement threshold, SMS information whether enabled or not and device GUI information. Workplace page includes the name of the workplace involved in the project. Subsequently, Order page contains the parameters of 'State Order', 'Monitoring State', 'Start monitoring', 'End monitoring' and 'Name of Workplace'. The 'Client page' contains all registered participants' devices information and finally the 'User page' provides the information about the current user who is using the system.

\subsection{System Architecture}

Figure 3 depicts the architecture of the overall InspectLife Surveillance and Telemonitoring system. The system houses all clinical data and notification events obtained from the smart phone device as described in the previous section. This information is available for: a) patients and their family care-givers; b) home care agency workers; c) medical practitioners; and d) operators providing system monitoring. All users are able to take an active part in responding to an emergency situation as detected and notified through the ISWS. The web system also provides access to de-identified patient data through separate researcher access privileges.

\section{$4 \quad$ Methodology}

A trial was developed to evaluate the effectiveness of the system in monitoring falls that targeted 100 senior citizens aged 65 and over living in the Australian Capital Territory (ACT) with either gait anomalies, a history of falls, or have a fear of falling. The duration of the pilot 


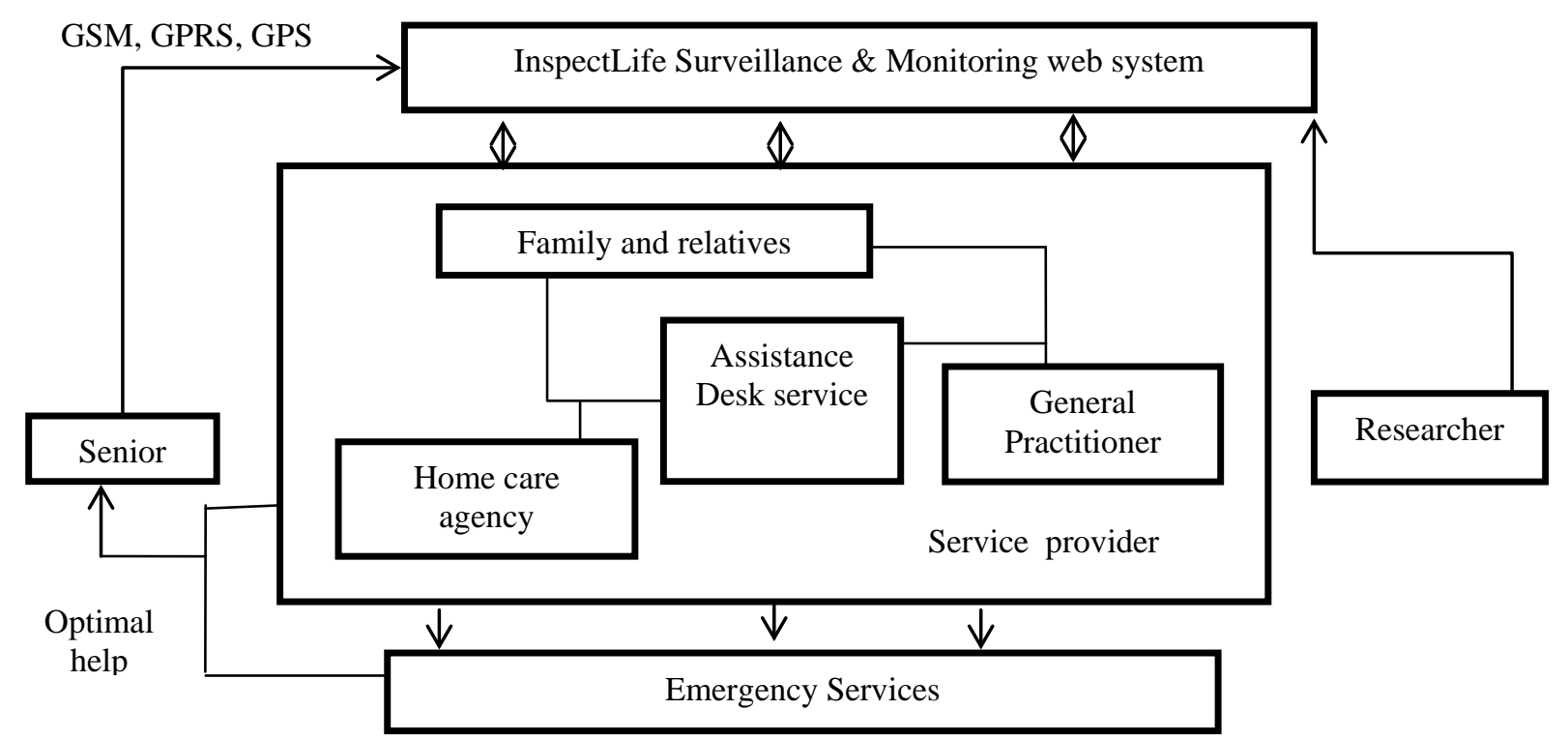

Figure 3: Enhanced Information system for Surveillance and Tele-monitoring based on Mediispect (Jansa, Zvolsky, Korec, Chod, \& Potucek, 2011)

program was six months and ran between May to October in 2012. Participants had the additional requirement of being able to live independently in the community and were recruited from clients enrolled in the Anglicare community care program. The Tele-health, Research \& Innovation Laboratory (THRIL), at UWS, prepared and configured 100 smart phones for the pilot program and hosted the ISWS servers to receive event information and raw tri-axial accelerometer data from the smart-phone devices. The mobile phones were distributed to participants who were trained on how to use the 'InspectLife' application. Access to general smart phone features and capabilities was disabled and participants wore the devices on their hip secured by a waistband.

All previous history of falls and other co-morbidities for each of the participants were recorded at the beginning of the program and inserted into the ISWS.

For the trial study, tri-axial accelerometer data transmitted from the smart phones was recorded and collated from the InspectLife web system (ISWS) and Raw Movement Data System (RMDS) for data analysis purposes. Occasionally it was necessary to manually retrieve data from smart phones through direct SD card access, due to poor reception or other networking issues associated with particular mobile phones. Some falls information was also collected from participants over telephones or physical contact as occasionally falls were sustained without wearing smart phones.

In order to assess the feasibility of developing a threshold based fall detection algorithm for the data obtained from this pilot study, the data was downloaded as comma separated values (csv) files and imported into Matlab. Scripts were developed to read tri-accelerometer data and output features such as the sum vector (SV), velocity (v) and changes in posture angle $\left(\Delta \theta_{z}\right)$ with the following formulae:

$$
\begin{aligned}
& S V=\sqrt{x^{2}+y^{2}+z^{2}} \\
& v=\int(S V-g) d t \\
& \Delta \theta_{z}=\cos ^{-1}\left(\vec{g}_{\text {seg, }}(t)\right)\left(\frac{180}{\pi}\right)-\cos ^{-1}\left(\vec{g}_{s e g, z}(t-1)\right)\left(\frac{180}{\pi}\right)
\end{aligned}
$$

Formulae for calculating velocity and changes in posture angle were obtained from (Bourke, et al., 2010). X, y, and $\mathrm{z}$ refer to the three axes along which tri-axial accelerometer data is recorded. G refers to one $g$ of acceleration, which is equal to approximately $9.81 \mathrm{~m} / \mathrm{s}^{2}$.

In addition, inputs to the script allowed a window size to be set. A window size of 100 data points, or 6 seconds, was used. Each window overlapped with half of the following window, allowing for $100 \%$ overlap. This ensured that events that fell on the border of windows would be adequately recorded. Features that were recorded from windows included the variance, mean, range, maximum and minimum of each window.

\section{Result}

\subsection{Recruitment Numbers}

The aim was to recruit one hundred senior participants for the pilot, but this target could not be fulfilled for a variety of reasons. After initial recruitment, forty-one participants were unable to either commence the program or participate for the duration of the pilot program. Table 1 and Figure 4 illustrate the distribution of participants by age group and gender that fall under this category. Overall, 10 male and 31 female participants terminated prematurely from the study, and were predominantly in the age group between 85-89. The main reasons for participants leaving the pilot program are listed in Table 2. 


\begin{tabular}{|l|l|l|l|l|l|l|l|l|}
\hline Age group & $\begin{array}{l}\text { DG } \\
\mathbf{H}^{\mathbf{a}}\end{array}$ & $\begin{array}{l}\text { Psy. } \\
\mathbf{D}^{\mathbf{b}}\end{array}$ & $\begin{array}{l}\mathbf{D} \\
\mathbf{D}^{\mathbf{c}}\end{array}$ & $\begin{array}{l}\text { CN } \\
\mathbf{C T}^{\mathbf{d}}\end{array}$ & $\begin{array}{l}\text { DPP } \\
\mathbf{C G}^{\mathbf{e}}\end{array}$ & $\begin{array}{l}\mathbf{N W} \\
\mathbf{C}^{\mathbf{f}}\end{array}$ & Deceased $^{\mathbf{g}}$ & \\
\hline$(<70)$ & 2 & 0 & 0 & 0 & 1 & 2 & 0 & Total \\
\hline$(70-74)$ & 3 & 0 & 1 & 1 & 1 & 1 & 0 & 5 \\
\hline$(75-79)$ & 0 & 1 & 0 & 2 & 1 & 1 & 0 & 7 \\
\hline$(80-84)$ & 5 & 1 & 2 & 1 & 0 & 0 & 0 & 5 \\
\hline$(85-89)$ & 6 & 0 & 4 & 3 & 3 & 0 & 0 & 9 \\
\hline$(90-94)$ & 3 & 0 & 1 & 4 & 1 & 0 & 0 & 16 \\
\hline$(>95)$ & 0 & 0 & 0 & 0 & 0 & 0 & 1 & 8 \\
\hline Total & 19 & 2 & 8 & 11 & 7 & 4 & 1 & 51 \\
\hline
\end{tabular}

Table 1: Reasons for early termination from the falls study

${ }^{a}$ deterioration of general health (DGH)

${ }^{\mathrm{b}}$ psychiatric deterioration (Psy. D)

${ }^{\mathrm{c}}$ deepening of dementia (DD)

${ }^{\mathrm{d}}$ could not cope with technology (CNCT)

${ }^{\mathrm{e}}$ deterioration in health of the person for whom they are primary care giver (DPPCG)

${ }^{\mathrm{f}}$ not willing to continue with trial (NWC)

g deceased.

\begin{tabular}{|l|r|r|l|}
\hline $\begin{array}{l}\text { Age } \\
\text { group }\end{array}$ & Male & Female & \multicolumn{1}{l|}{ Participants } \\
\hline$(<70)$ & 0 & 4 & 4 \\
\hline$(70-74)$ & 1 & 4 & 5 \\
\hline$(75-79)$ & 0 & 6 & 6 \\
\hline$(80-84)$ & 2 & 5 & 7 \\
\hline$(85-89)$ & 5 & 7 & 12 \\
\hline$(90-94)$ & 1 & 5 & 6 \\
\hline$>94$ & 1 & 0 & 1 \\
\hline \multicolumn{1}{|c|}{ Total } & 10 & 31 & 41 \\
\hline
\end{tabular}

Table 2: Number of participants terminating early from the falls study by age group

\subsection{Technical issues with the Smart phones}

From the group of 57 participants who were able to participate for the duration of the study, Figure 4 further illustrates some of the technical issues encountered in collecting data from their active mobile devices. Six devices were unable to send any data to central servers. Out of the remaining 51 mobile devices that were able to send data, 29 mobile devices sent data packets regularly and consistently throughout the study whereas 22 devices

were only irregularly sending information. From the latter group, data losses occurred at different stages of the pilot. The reasons for irregular data being received are not clearly known. Possible reasons include poor network connection or a software bug in the application software that would inadvertently disable the network connection. For devices that had significant problems in transmitting data, data could alternatively be retrieved manually by directly reading the SD cards contained within each smart phone on a regular basis.

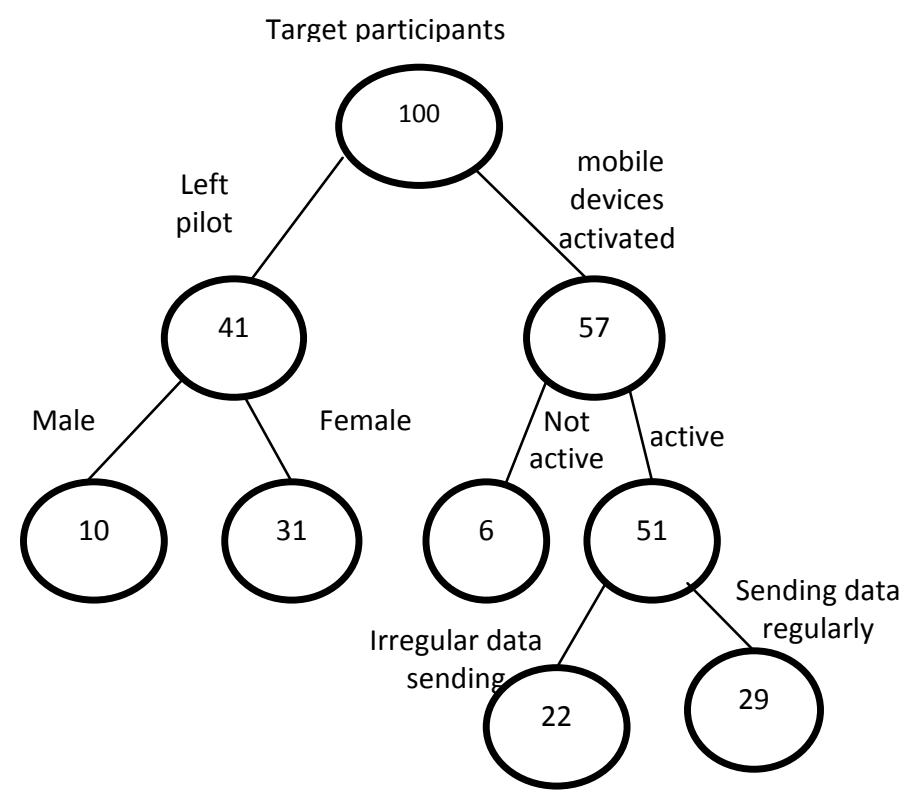

Figure 4: Participants and mobile device category 


\subsection{Falls captured by the Smart phones}

There are many 'Possible Falls' were captured from the smart phones during the trial. An operator contacted the subject to verify whether a fall has occurred whenever a 'Possible Fall' signal arrives in the ISWS. In the case that a fall was verified to have occurred, the occurrence was recorded in the ISWS, and the operator took the necessary action to ensure the safety of the subject.

Table 3 presents the comparison of falls and hospitalisation occurred with a non-intervention group, mainly acquired from past hospital records for an

\begin{tabular}{|l|l|l|l|l|}
\hline & $\begin{array}{l}\text { Non- } \\
\text { interventi } \\
\text { on } \\
\text { group: } \\
2007 / 200 \\
8\end{array}$ & $\begin{array}{l}\text { Non- } \\
\text { interventi } \\
\text { on } \\
\text { group: } \\
2011\end{array}$ & $\begin{array}{l}\text { Interve } \\
\text { ntion } \\
\text { group: } \\
2011\end{array}$ & $\begin{array}{l}\text { Intervent } \\
\text { ion } \\
\text { group:20 } \\
12\end{array}$ \\
\hline Fell & $26.6 \%$ & $68 \%$ & $33 \%$ & $22 \%$ \\
\hline $\begin{array}{l}\text { Major } \\
\text { falls } \\
\text { (hospit } \\
\text { alised) }\end{array}$ & $2.30 \%$ & $2 \%$ & $8 \%$ & $2 \%$ \\
\hline
\end{tabular}

Table 3. Comparison of falls and hospitalization based on past hospital record

equivalent period to the trial in the previous year (April to October 2011). As a result, the total number of falls is $50 \%$ lower than the proportion of participants who sustained a fall in the non-intervention group for the previous year. The hospitalisation of falls during the trial was similar in proportion to the non-intervention group in 2011. Due to the small number of detected falls the differences in number of falls between both groups is not significant (Huq, Basilakis, \& Maeder, 2013).

\subsection{Captured falls from smart phone}

There are 456 possible falls had captured from the smart phone (Pirnejad, Huq, Basilakis, \& Maeder, 2014). Among these we can see that one subject had 224 possible falls and this subject has suffering from Hemiplegia in the left side. Next one subject had 62 possible falls, another subject had 36 possible falls, then one subject had 31 possible falls, two subjects had 25 possible falls, another subject had 22 possible falls, one subject had 14 possible falls; then next subject had 10 possible falls records. There are 8 times, 6 times, 5 times possible falls occurred for two subjects each. One subject had 4 possible falls, six subjects had 3 possible falls, then 13 subjects had 2 possible falls and finally 37 subjects had one possible falls. The high number of falls detected leads to a low specificity, showing that the simple threshold based system used in the study is not sufficient as an effective fall detection system.

\subsection{Real falls}

There were six participants who fell during the pilot program who were wearing their fall monitoring devices at the time of the fall. Among them, two participants fell five times each. There were no hospitalisations as a result of these falls. There were also eight falls from six participants although they were not using their devices at the particular time. Two falls in this category resulted in the participants being hospitalized.

\subsection{Survey result from the participants}

A survey was conducted for the participants at the end of the pilot program, of which some of the results are summarized in Figure 5. The majority of the participants responded in the positive that: a) they were contacted often by the operator for assistance or monitored continuously; b) they were not limited in their daily activities (DA) when wearing the device; c) they were overall satisfied with the device used in the pilot program; f) they felt comfortable that activity data was collected and sent to the health care team. On the other hand, a majority of participants did not feeling safer whilst wearing the device and did not find the device easy to understand. In addition, a few of the participants also commented and expressed fear that they would not be able to live independently despite having 24 hours monitoring, available. Majority of them also commented and expressed discomfort at the idea of having both a mobile phone and falls detecting device together in one smart phone; preferring only one function at a time instead of multifunctional device.

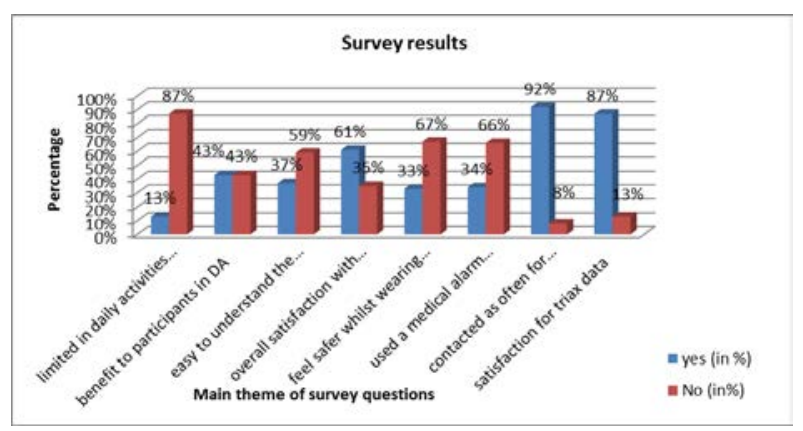

Figure 5: Survey results from the participants

\subsection{Feasibility of threshold-based algorithm using data obtained}

When a fall occurs, the sum vector will first decrease to close to zero, reflecting that the body is in free fall. Upon impact, the sum vector will increase markedly, to several $g$ s of acceleration. After this, the SV will oscillate as it returns to a value of $1 \mathrm{~g}$. This simple fall model can be used to develop hypotheses for how the 5 features used will be affected if a fall occurs. However, this fall model is highly simplistic, as it assumes that before, during, and after the fall, no extraneous movement occurs. As such, fall models of greater complexity should be considered to consider other types of fall.

If an entire window is able to encompass a fall, as would be expected with the current overlapping windowing regime, the five features are hypothesised to behave in unique ways. Although some of the features may provide similar readings to high energy activities such as running, when combined, all of the features should be able to differentiate between falls and other activities. Table 4 expresses the expected changes in the five features during a fall. 


\begin{tabular}{|l|l|}
\hline \multicolumn{1}{|c|}{ Feature } & \multicolumn{1}{|c|}{ Expected Behaviour } \\
\hline Variance & $\begin{array}{l}\text { Should increase as there will be more } \\
\text { accelerometer data recordings further } \\
\text { away from the mean. }\end{array}$ \\
\hline Mean & $\begin{array}{l}\text { May change from the expected } 1 \mathrm{~g} \text { value } \\
\text { that would be measured when at rest, } \\
\text { however this may not always happen. }\end{array}$ \\
\hline Range & $\begin{array}{l}\text { Will increase, as the maximum will be } \\
\text { very high and the minimum will be close } \\
\text { to zero. }\end{array}$ \\
\hline Minimum & \begin{tabular}{l} 
Should increase to several $g$ s. \\
\hline Maximum
\end{tabular} Should decrease to close to zero. \\
\hline
\end{tabular}

Table 3: Expected changes in features during a fall

Fall data from one of the subjects, is shown below in Figure 6.
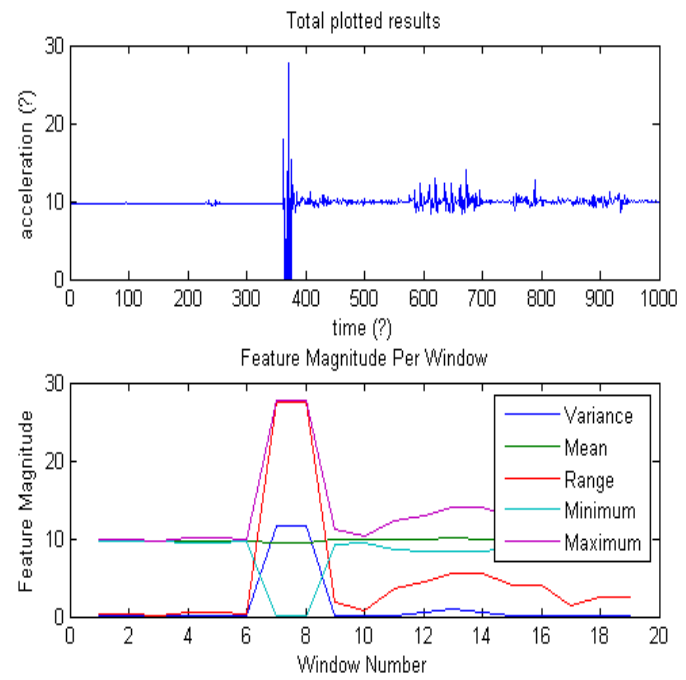

Figure 6: Fall data from one of the subjects with window based features

Variance is observed to significantly increase for the windows which contain the fall, as predicted. Range, Minimum, and Maximum also change as expected. No significant change is observed for Mean, suggesting that Mean is not a discriminating feature for falls.

Fall data for another subject, measured on the $27^{\text {th }}$ of September 2012, is shown below in Figure 7.
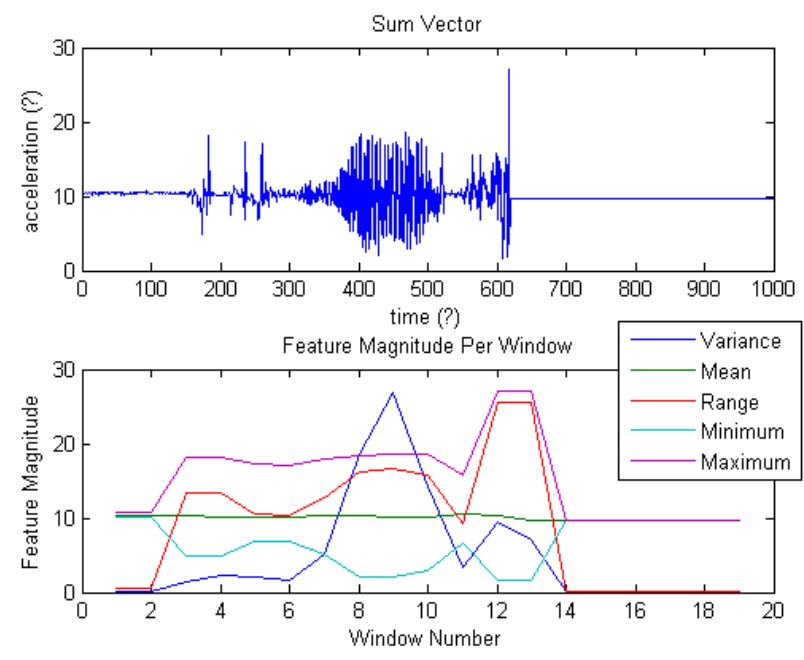

Figure 7: Fall data from another subject with window based features

The fall was detected at the peak seen shortly after the $600^{\text {th }}$ data point above. Strangely, after the peak, the accelerometer detected almost no changes in acceleration, as seen in the sum vector graph above. This suggests that the fall occurred earlier than detected, and that the sudden impact that was detected may have taken place after the fall. This highlights the unpredictable nature of real world falls. An increased sample rate (greater than the $16.67 \mathrm{~Hz}$ used here) may allow for better detection of falls.

Fall data for another subject, measured on the $9^{\text {th }}$ of September 2012, is shown below in Figure 8.
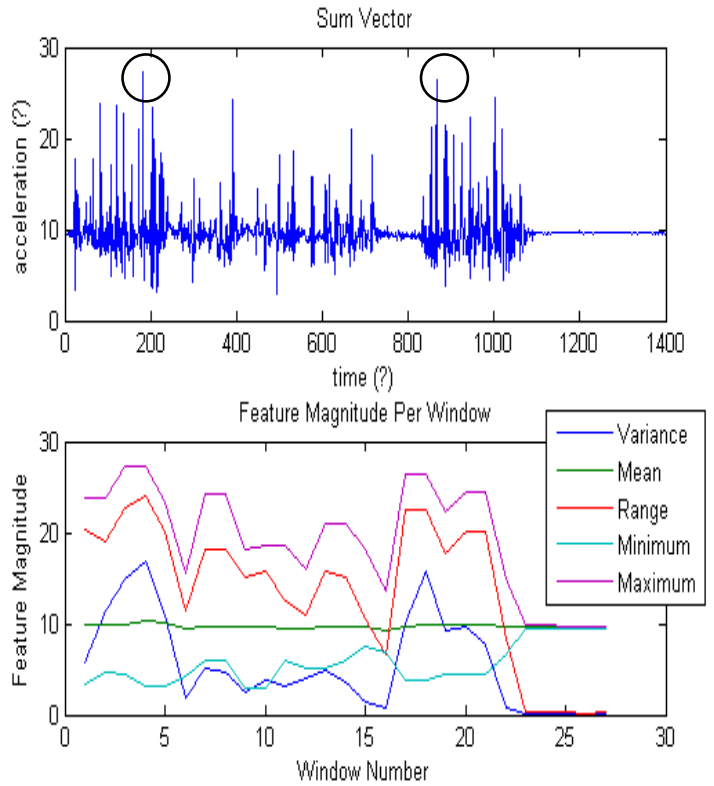

Figure 8: Fall data from another subject with window based features and detected falls indicated 
Two falls were detected, as marked by the circles. The first detected fall was an actual fall, whereas the second was reported as a non-fall. As seen in the features graph, these two peaks, as well as other peaks through the acceleration data give readings suggesting falls have occurred multiple times, despite only happening once. This is likely due to unsteadiness on the part of the end user in this case.

Velocity data was obtained from data obtained through same subject, on the $14^{\text {th }}$ of June 2012. This data is graphed with the SV - g in Figure 9. The value for g was taken at $9.81 \mathrm{~m} / \mathrm{s}^{2}$. However, as can be seen, velocity climbs more or less continuously to very high values during activity and comes down during periods of relative inactivity.

During an activity, velocity is expected to remain less than $10 \mathrm{~m} / \mathrm{s}$. Velocity is expected to fall to zero once the activity ends. As such, it would be expected that a sum of the acceleration undertaken during an activity would total zero, which would give a final velocity equal to the initial velocity. This is not observed in the velocity data. A possible reason for this may be that the gravitational acceleration value selected is not high enough.

However, it is also noticed that during periods of inactivity, when no changes in velocity should occur, the velocity decreases. This may be caused by the value of gravitational acceleration selected not being low enough. It is thus apparent that these two issues cannot be solved simply by changing the value of gravitational acceleration selected.
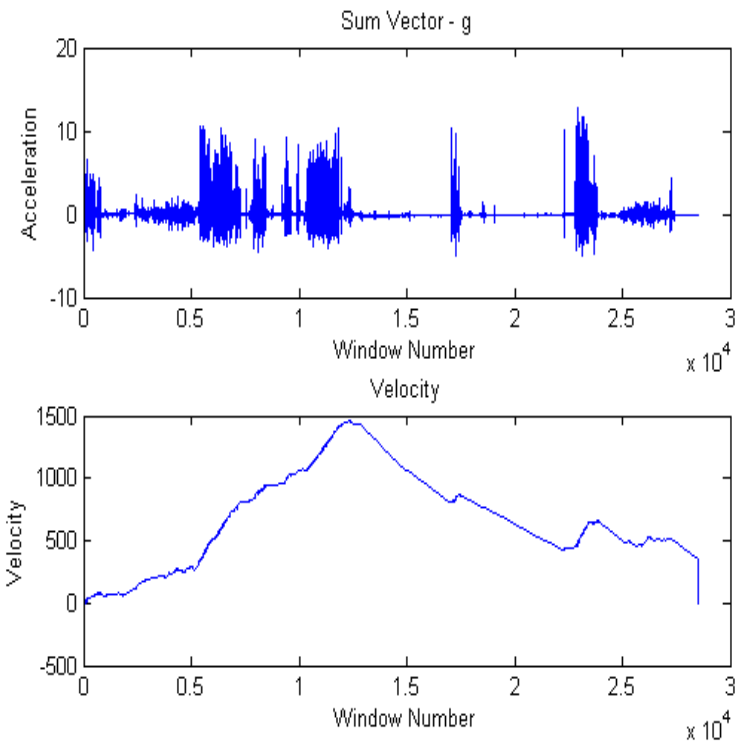

Figure 9: Velocity data calculated from data recorded by device 735

The likely causes of this issue are:

1) The sampling rate is not sufficient to pick up enough negative acceleration data points.

2) The accelerometer requires zeroing once and only once in order to pick up the correct value for gravity.

The $2^{\text {nd }}$ issue can be temporarily corrected when scripting by choosing the value for gravitational acceleration as the median of the SV array as long as the data has only been zeroed once throughout the time of recording.

Posture angle and posture changes have been recorded in Figure 10 from a section of the data recorded by same subject on the $14^{\text {th }}$ of June 2012. A cursory examination of the results obtained for posture changes suggests that the posture data obtained is valid and should be usable for threshold based fall detection. Further examination of change in posture angle will necessary if this feature is to be used as part of a threshold based fall detection algorithm.
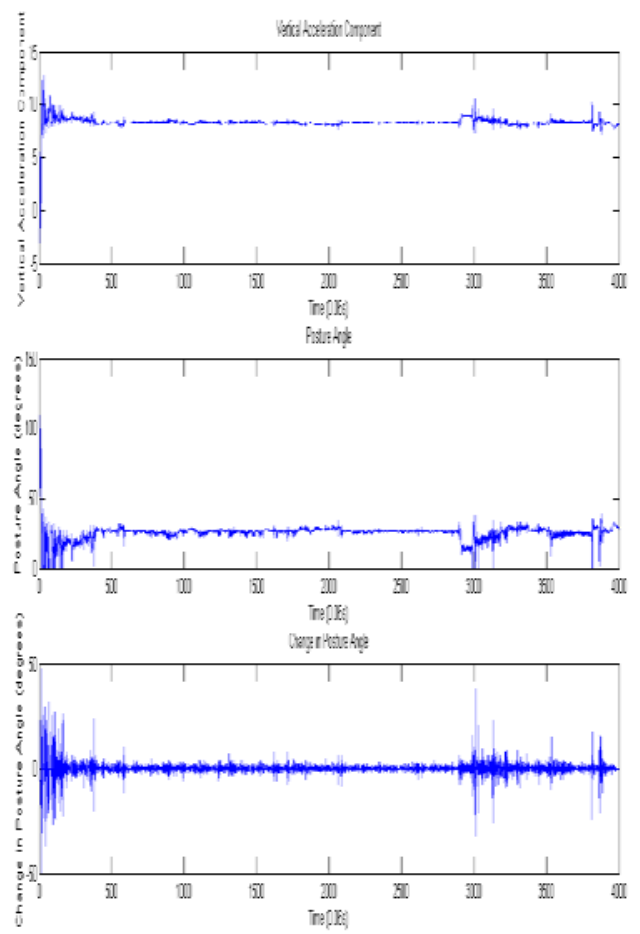

Figure 50: Changes in posture angle

\section{Discussion}

The pilot program has provided real time experience about various issues regarding fall-detection devices used by the elderly. For example, even though 100 elderly were targeted for this program only a moderate fraction of these participants participated, with approximately half of the participants terminating due to various reasons at the very start of the program for various reasons. Consequences have been minimal for falls sustained that have been captured by the device. No participants required hospitalisation. But some of the falls also sustained without the device and the consequences in this category of falls were significant. That is, some of them were hospitalised. Therefore we can predict that monitoring by means of wearing the device is potential to reduce the risk of fall.

It is also observed that the elderly feel that they are cared for by somebody, which can be of great significance to the subjects in certain cases. A sense of security is provided in that one knows one is being monitored. As fear of fall and for their safety are two major reasons for the elderly to enter aged care facilities, 
if they can use supports such as the mobile devices used in this trial then they can remain in their own home or self-care unit easily to ensure peace of mind.

Through initial analysis of accelerometer data gained from this study to test the feasibility of building a fall detection algorithm from the acquired data, it has been determined that there are various issues with data obtained. This data is largely not of sufficient quality to allow for threshold based fall detection algorithms to detect falls with a high sensitivity and low rate of false alarms. The biggest issues are with the data's low sampling rate of $16.67 \mathrm{~Hz}$ and low range of $\pm 2 \mathrm{~g}$. This leads to inaccuracies and saturation of the acceleration vectors, which cause difficulty in determining thresholds for the sum vector and the window based features. In addition, accelerometers must be zeroed only once before recording to allow vertical velocity to be effectively determined. Fall data obtained is sufficient to detect changes in posture, suggesting that changes in posture will be a vital tool when a fall detection algorithm is constructed.

\section{Conclusions}

An overview of a pilot study of fall prevention among the elderly has been presented and results gained have been discussed. Positive results gained been gained from the pilot study itself, with no serious falls occurring for elderly individuals wearing the device. Participants also felt that they were cared for during the study, giving them peace of mind and a sense of security. Study of the accelerometer data gained during the study has shown that data collected is largely not of sufficient quality for an effective threshold based fall detection algorithm to be put in place. Both the sample rate and range within which data was recorded should be improved. However, the data was of sufficient quality for changes in posture to be detected in a reasonable manner, opening the door for algorithms using this feature. Further study is to be done to construct an algorithm to detect falls using this data, as well as higher quality data obtained in the future.

\section{Acknowledgement}

The trial was supported by the ACT Government (Australia) under the ACT Health Promotion Grants Program.

\section{References}

Bagalà, F., Becker, C., Cappello, A., Chiari, L., Aminian, K., Hausdorff, J. M., et al. (2012). Evaluation of Accelerometer-Based Fall Detection Algorithms on Real-World Falls. PLoS ONE , 7 (5).

Bianchi, F., Redmond, S., Narayanan, M., Cerutti, S., \& Lovell, N. (2010). Barometric Pressure and Triaxial Accelerometry-Based Falls Event Detection. Neural Systems and Rehabilitation Engineering, IEEE Transactions on , 18 (6), 619-627.

Bourke, A., O'Brien, J., \& Lyons, G. (2007). Evaluation of a threshold-based tri-axial accelerometer fall detection algorithm. Gait \& Posture , 26 (2), 194-199.

Bourke, A., van de Ven, P., Gamble, M., O'Connor, R., Murphy, K., Bogan, E., et al. (2010). Assessment of waist-worn tri-axial accelerometer based fall-detection algorithms using continuous unsupervised activities. Engineering in Medicine and Biology Society (EMBC), 2010 Annual International Conference of the IEEE (pp. 2782-2785). Buenos Aires: IEEE.

Boyle, J., \& Karunanithi, M. (2008). Simulated fall detection via accelerometers. Engineering in Medicine and Biology Society, 2008. EMBS 2008. 30th Annual International Conference of the IEEE (pp. 1274-1277). Vancouver, BC: IEEE.

Dai, J., Bai, X., Yang, Z., \& Shen, Z. X. (2010). Mobile phone-based pervasive fall detection. Personal and Ubiquitous Computing , 14 (7), 633-643.

Dumitrache, M., \& Pasca, S. (2013). Fall detection algorithm based on triaxial accelerometer data. EHealth and Bioengineering Conference (EHB) (pp. 14). Lasi: IEEE.

Freescale Semiconductor. (2013). Xtrinsic MMA8451Q 3Axis, 14-bit/8-bit Digital Accelerometer. Retrieved July 22, 2014, from Freescale: http://cache.freescale.com/files/sensors/doc/data_sheet/ MMA8451Q.pdf

Guest, C., Halliday, L., Kee, C., O'Brien, E., \& O'Donoughue, R. (2010). Australian Capital Territory: Chief Health Officer's Report 2010. Retrieved July 12, 2014, from ACT Health: http://health.act.gov.au/publications/reports/chiefhealth-officer-reports/chief-health-officers-report-2010

Huq, G., Basilakis, J., \& Maeder, A. (2013). Evaluation of a fall intervention pilot program for the elderly at risk of falls: Surveillance and telemonitoring via smart phone tri-axial accelerometery. International Conference on Successes and Failures in Telehealth, 4th Annual Meeting of the Australasian Telehealth Society. Brisbane.

Jansa, J., Zvolsky, K., Korec, J., Chod, J., \& Potucek, J. (2011). Czech pHealth Projects 2011. Retrieved July 12, 2014, from ITU Telecom World: http://www.ituworld.cz/download/presentations/6Jansa_pHealt_projects_2011.pdf

Jantaraprim, P., Phukpattaranont, P., Limsakul, C., \& Wongkittisuksa, B. (2010). Improving the Accuracy of a Fall Detection Algorithm Using Free Fall Characteristics. Electrical Engineering/Electronics Computer Telecommunications and Information Technology (ECTI-CON), 2010 International Conference on (pp. 501-504). Chiang Mai: IEEE.

Jia, N. (2009). Detecting Human Falls with a 3-Axis Digital Accelerometer. Analog Dialogue , 43 (7), 1-7.

Kangas, M., Konttila, A., Winblad, I., \& Jämsä, T. (2007). Determination of simple thresholds for accelerometry-based parameters for fall detection. Conf Proc IEEE Eng Med Biol Soc (pp. 1367-1370). IEEE.

Kangas, M., Vikman, I., Nyberg, L., Korpelainen, R., Lindblom, J., \& Jämsä, T. (2012). Comparison of reallife accidental falls in older people with experimental falls in middle-aged test subjects. Gait Posture , 35 (3), 500-505.

Kangas, M., Vikman, I., Wiklander, J., Lindgren, P., Nyberg, L., \& Jämsä, T. (2009). Sensitivity and 
specificity of fall detection in people aged 40 years and over. Gait \& Posture , 29 (4), 571-574.

Karantonis, D., Narayanan, M., Mathie, M., Lovell, N., \& Celler, B. (2006). Implementation of a real-time human movement classifier using a triaxial accelerometer for ambulatory monitoring. Information Technology in Biomedicine, IEEE Transactions on. 10, pp. 156-167. IEEE.

Kerdegari, H., Samsudin, K., Ramli, A., \& Mokaram, S. (2012). Evaluation of fall detection classification approaches. Intelligent and Advanced Systems (ICIAS), 2012 4th International Conference on. 1, pp. 131-136. Kuala Lumpur: IEEE.

Klenk, J., Becker, C., Lieken, F., Nicolai, S., Maetzler, W., Alt, W., et al. (2011). Comparison of acceleration signals of simulated and real-world backward falls. Med Eng Phys , 33 (3), 368-373.

Lord, S. R., Sherrington, C., \& Menz, H. B. (2001). Falls in older people: risk factors and strategies for prevention. : Cambridge University Press.

Nyan, M., Tay, F., Seah, K., \& Sitoh, Y. (2006). Classification of gait patterns in the time-frequency domain. Journal of Biomechanics , 39 (14), 2647-2656.

Pérolle, G. (2006). Automatic fall detection and activity monitoring for elderly. Retrieved July 12, 2014, from Medetel:

http://www.medetel.lu/download/2006/parallel_session s/presentation/0407/Perolle.pdf

Pirnejad, H., Huq, G., Basilakis, J., \& Maeder, A. (2014). Monitoring Falls in Elderly People: Lessons from a Community-based Project, 3rd International Conference on Global Telehealth Durban, South Africa: Studies in Health Technology and Informatics

Sorvala, A., Alasaarela, E., Sorvoja, H., \& Myllyla, R. (2012). A two-threshold fall detection algorithm for reducing false alarms. Medical Information and Communication Technology (ISMICT), 2012 6th International Symposium on (pp. 1-4). La Jolla, CA: IEEE.

World Health Organization. (2013). Ageing and Life Course. Retrieved July 12, 2014, from World Health Organization: http://www.who.int/ageing/en/

World Health Organization. (2012). Falls: Fact Sheet No. 344. Retrieved July 12, 2014, from World Health Organization:

http://www.who.int/mediacentre/factsheets/fs344/en/

World Health Organization. (2007). WHO global report on falls prevention in older age. Retrieved July 14, 2014, from World Health Organization: http://www.who.int/violence_injury_prevention/public ations/other_injury/falls_prevention.pdf

Zhao, Z., Chen, Y., Wang, S., \& Chen, Z. (2012). FallAlarm: Smart Phone Based Fall Detecting and Positioning System. Procedia Computer Science , 10, 617-624. 